14. Ungchusak K, Auewarakul P, Dowell SF, et al. Probable person-to-person transmission of avian influenza (H5N1). N Engl J Med 2005; 352:333-340.

\section{Prevalence of Hepatitis B and C Among Brazilian Dentists}

TO THE EDITOR-There is great concern about transmission of bloodborne pathogens in dentistry ${ }^{1-2}$ because dentists work with limited access and restricted visibility and frequently use aerosol-forming equipment and sharp devices. ${ }^{3}$ Accordingly, we conducted a serologic survey of hepatitis $B$ and $C$ status among dentists working in a town in the state of São Paulo in Brazil from August 2001 to April 2002. All dentists currently working in the city of Sertãozinho were contacted by telephone, always by the same investigator, who scheduled an individual meeting for a personal interview and collection of a blood sample. Of the 147 dentists contacted, 12 refused to participate in the study because it involved blood collection, resulting in a study population of 135 dentists.

Dentists were asked about previous hepatitis $B$ vaccination, whether their main affiliation was a public or a private service, and the duration of their professional practice. A $10-\mathrm{mL}$ blood sample was collected from each participant. Enzymelinked immunosorbent assay testing was done in according to manufacturer instructions. We tested serum samples for the presence of hepatitis B surface antigen ( $\mathrm{HBsAg}$ ), antibody to hepatitis B surface antigen (anti-HBsAg), antibody to hepatitis $\mathrm{B}$ core antigen (anti-HBcAg) immunoglobulin $\mathrm{G}$ (with the Hepatonostika test; Organon Teknika), and antibody to hepatitis $\mathrm{C}$ virus (anti-HCV; with the Ortho HCV 3.0 ELISA; Organon Teknika). Serum samples that tested positive for $\mathrm{HBsAg}$ were further evaluated for the presence of hepatitis $\mathrm{B} e$ antigen (HBeAg), and anti-HBeAg (Abbott) and samples positive for anti-HCV were also tested for the presence of HCV RNA by polymerase chain reaction (PCR) (Amplicor; Roche Diagnostics).

Mean age of the study population was 34.5 years (range, 22-56 years), and the sex distribution showed a slight predominance of women ( $63.0 \%$ female). Most of the dentists $(77.0 \%)$ had a private practice as their main professional occupation, and the other $23.0 \%$ had a public service as their main affiliation. Among the dentists enrolled, $70.4 \%$ had been practicing dentistry for 14 years or less, and $5.2 \%$ had been working for more than 25 years.

One dentist $(0.7 \%)$ tested positive for HBsAg. The same dentist tested positive for anti-HBeAg and tested negative for $\mathrm{HBeAg}$. Anti-HBsAg and anti-HBcAg antibodies were detected in serum samples from $11(8.1 \%)$ of the dentists, and $99(73.3 \%)$ tested positive only for anti-HBsAg. The other 24 dentists (17.9\%) showed no positive serologic markers for HBV. Table 1 summarizes the distribution of HBV serologic markers according to age and length of professional practice.
TABLE. Prevalence of Serologic Markers for Hepatitis B Virus Among the 135 Dentists in the Study, According to Age and Duration of Professional Practice

\begin{tabular}{lccc}
\hline & \multicolumn{3}{c}{ Proportion (\%) of participants } \\
\cline { 2 - 4 } Variable & $\begin{array}{c}\text { Positive for } \\
\text { HBsAg }\end{array}$ & $\begin{array}{c}\text { Positive for } \\
\text { anti-HBcAg }\end{array}$ & $\begin{array}{c}\text { Positive for } \\
\text { anti-HBsAg }\end{array}$ \\
\hline $\begin{array}{c}\text { Age range in years } \\
\text { 20-29 }\end{array}$ & 0 & 0 & $37 / 39(94.9)$ \\
$30-39$ & 0 & $3 / 61(4.9)$ & $50 / 61(82.0)$ \\
$40-49$ & $1 / 28(3.6)$ & $6 / 28(21.4)$ & $19 / 28(67.9)$ \\
$50-59$ & 0 & $3 / 7(42.9)$ & $4 / 7(57.1)$ \\
Duration of practice & & & \\
in years & & & \\
$0-4$ & 0 & 0 & $29 / 30(96.7)$ \\
$5-9$ & 0 & $1 / 36(2.8)$ & $28 / 36(77.8)$ \\
$10-14$ & 0 & $3 / 29(10.3)$ & $25 / 29(86.2)$ \\
$15-19$ & 0 & 0 & $13 / 16(81.3)$ \\
$20-24$ & $1 / 17(5.9)$ & $4 / 17(23.5)$ & $10 / 17(58.8)$ \\
$25-30$ & 0 & $4 / 7(57.1)$ & $5 / 7(71.4)$ \\
\hline
\end{tabular}

NOTE. Each subject provided a single serum sample for testing. Anti$\mathrm{HbcAg}$, antibody to hepatitis B core antigen; anti-HbsAg, antibody to hepatitis B surface antigen; $\mathrm{HbsAg}$, hepatitis B surface antigen.

Only 1 dentist $(0.7 \%)$ tested positive for anti-HCV antibody. $\mathrm{HCV}$ infection was further confirmed by testing the same serum sample with PCR for HCV RNA, which also gave a positive result. This dentist also tested positive for anti$\mathrm{HBcAg}$ and anti-HBsAg.

When asked about receiving any dose of HBV vaccine, 133 $(98.5 \%)$ of the 135 dentists enrolled reported that they had received at least 1 dose of the vaccine. Most of those exposed to the HBV vaccine had been offered the vaccine by the local public health system $(50.4 \%)$ or by their dental school $(31.6 \%)$. Only $18.0 \%$ of participants had spontaneously sought vaccination at private clinics. Among those vaccinated with at least 1 dose, only $17(12.8 \%)$ of 133 reported that they had undergone a postvaccination anti-HBsAg test to confirm immunization against $\mathrm{HBV}$. The rates of seroconversion to anti-HBsAg among vaccinated dentists, excluding those with evidence of natural immunization (ie, those who were anti-HBcAg positive) were as follows: 2 (50.0\%) of 4 participants who reported receiving 1 dose of vaccine, 7 (100.0\%) of 7 participants who reported receiving 2 doses, and $90(81.1 \%)$ of 111 participants who reported receiving 3 or more doses.

The serologic test results were personally delivered by the investigator to the participating dentists, with a letter of orientation about hepatitis B and C, occupational risk, and measures to prevent acquisition of pathogens during professional practice. Those with evidence of active hepatitis $\mathrm{B}$ or $\mathrm{C}$ infection were asked to attend a medical consultation at a reference university hospital.

Before the vaccine against HBV became available in the 1980s, the seroprevalence of HBV was higher among dentists than in the general population. ${ }^{4}$ In view of the importance 
of vaccination against $\mathrm{HBV}$ among health professionals, ${ }^{1-2}$ vaccination campaigns by professional organizations and public institutions in Brazil and in other countries have contributed to an increase in compliance with vaccination among dentists. $^{4-5}$

Brazilian dentists were first motivated to receive vaccination against HBV by means of a campaign conducted by the Federal Dentistry Council in partnership with teaching institutions and public services in 1995 and again in 2002. The effect of these mobilizations was demonstrated in the present investigation by the high percentage of professionals who were vaccinated $(98.5 \%)$ and also by the high proportion of participants vaccinated through these entities $(82.0 \%)$.

Although vaccine against $\mathrm{HBV}$ is highly effective, a small proportion of subjects do not seroconvert after the first vaccination. In the present series, serologic testing revealed a higher-than-expected rate of vaccination failure among individuals who received the full course of vaccinations. Possible risk factors that prevent seroconversion were not analyzed, and the information reported by the participants was not confirmed by review of their vaccination cards.

The high rate of vaccination failure supports the need for postvaccination follow-up. In spite of the findings of other studies, ${ }^{6}$ we detected a very low rate of compliance with antiHBsAg testing, which may be explained by the unavailability of the test in the primary public healthcare network, the lack of a formal recommendation on the part of those who supply the vaccine, the high cost of the test in the private healthcare system, and lack of awareness on the part of the professionals regarding their risk of $\mathrm{HBV}$ acquisition.

The prevalence of active HBV or HCV infection detected in the present series was similar to that detected by other investigators among dentists ${ }^{7,8}$ and also similar to the that observed in São Paulo among the general population, ${ }^{9}$ although other Brazilian regions have higher prevalences of HBV infection. ${ }^{10}$ The rate of previous HBV infection identified was higher than that detected in São Paulo among the general population $(4.90 \%),{ }^{9}$ a fact possibly related to the occupational exposure of dentists to this virus, although other risk factors were not investigated. In the present study, the prevalence of anti-HBcAg positivity increased with age, and the prevalence also increased with the duration of professional practice, which may be explained by the cumulative risk of professional exposure to HBV acquisition.

In the present study, the individual who tested positive for anti-HCV had been working as a dentist for more than 20 years and was older than 40 years. These data agree with the literature and emphasize the cumulative risk of acquisition of HCV and/or HBV with increasing age and increasing length of professional practice. ${ }^{8}$

The present study alerts us to the need for dentists to undergo a test to confirm acquisition of immunity after vac- cination against hepatitis $B$, and the need to always use standard precautions during professional practice to avoid occupational acquisition of hepatitis B and C, among other diseases transmitted via the blood.

\section{Wanessa Teixeira Bellíssimo-Rodrigues, MS, DDS; Alcyone Artioli Machado, MD, PhD; Fernando Bellíssimo-Rodrigues, MD, MS; Margarida P. Nascimento; José Fernando C. Figueiredo, MD, PhD}

From the Program in Internal Medicine, Area of Biosciences Applied to Internal Medicine (W.T.B.-R.), the Area of Infectious and Parasitic Diseases, Department of Internal Medicine (A.A.M., J.F.C.F.), and the Committee for the Control of Hospital Infections (F.B.-R.) and the Program in Biomedicine, Laboratory of Serology (M.P.N.), University Hospital, Faculty of Medicine of Ribeirão Preto, University of São Paulo, Brazil.

Address reprint requests to Alcyone A. Machado, MD, Department of Internal Medicine, University Hospital, Faculty of Medicine of Ribeirão Preto, USP Avenida dos Bandeirantes, 3900, Campus Universitário Monte Alegre, 14048-900, Ribeirão Preto, São Paulo, Brasil (aamachad@fmrp.usp.br. or wanessat@ig.com.br).

Infect Control Hosp Epidemiol 2006; 27:887-888

(C) 2006 by The Society for Healthcare Epidemiology of America. All rights reserved. 0899-823X/2006/2708-0019\$15.00.

\section{REFERENCES}

1- Kohn WG, Collins AS, Cleveland JL, Harte JA, Eklund KJ, Malvitz DM, Centers for Disease Control and Prevention (CDC). Guidelines for infection control practices in dental health-care settings. MMWR Recomm Rep 2003; 52(RR-17):1-61.

2. Cottone JA, Terezhalmy GT, Molinari JA. Practical Infection Control in Dentistry. 2nd ed. Philadelphia: Williams \& Wilkins; 1996:437.

3. Prospero E, Savini S, Annino I. Microbial aerosol contamination of dental healthcare workers' faces and other surfaces in dental practice. Infect Control Hosp Epidemiol 2003; 24:139-141.

4. Cleveland JL. Hepatitis B vaccination and infection among U.S. dentists, 1983-1992. J Am Dent Assoc 1996; 127:1385-1390.

5. Martins AM, Barreto SM. Hepatitis B vaccination among dentists. Rev Saúde Pública 2003; 37:333-338.

6. Mccarthy GM, Koval JJ, Macdonald JK. Occupational injuries and exposures among Canadian dentists: the results of a national survey. Infect Control Hosp Epidemiol 1999; 20:331-336.

7. Ammon A, Reichart PA, Pauli G, Petersen LR. Hepatitis B and C among Berlin dental personnel: incidence, risk factors, and effectiveness of barrier prevention measures. Epidemiol Infect 2000; 125:407-413.

8. Weber C, Schaub DC, Fried R, Lambrecht IT, Erb P, Meyer J. Low prevalence of hepatitis $\mathrm{C}$ virus antibody among Swiss dental health care workers. J Hepatol 2001; 34:963-967.

9. Foccacia R, Conceição OJG da, Sette JR, et al. Estimated prevalence of viral hepatitis in the general population of the municipality of São Paulo, measured by serologic survey of a stratified, randomized and residencebased population. Braz J Infect Dis 1998; 2:269-284.

10. Tavares-Neto J, Almeida D, Soares MC, et al. Seroprevalence of hepatitis $B$ and $C$ in the western Brazilian Amazon region (Rio Branco, Acre): a pilot study carried out during a hepatitis $B$ vaccination program. Braz $J$ Infect Dis 2004; 8:133-139. 